\title{
Duhuo Jisheng Decoction inhibits SDF-1-induced inflammation and matrix degradation in human degenerative nucleus pulposus cells in vitro through the CXCR4/NF-kB pathway
}

\author{
Zong-chao LIU ${ }^{1, *, \#}$, Zhen-long WANG ${ }^{1, \#}$, Chen-yi HUANG ${ }^{1}$, Zhi-jiang FU ${ }^{1}$, Yong LIU ${ }^{1}$, Zhang-chao WEI ${ }^{1}$, Shi-gui LIU ${ }^{1}$, Chuan \\ $\mathrm{MA}^{1, *}$, Jie-liang SHEN ${ }^{2}$, Dayue Darrel DUAN ${ }^{3,4}$ \\ ${ }^{1}$ Department of Orthopedic Surgery, the Traditional Chinese Medicine Affiliated Hospital of Southwest Medical University, Luzhou \\ 646000, China; ${ }^{2}$ Department of Orthopedic Surgery, the First Affiliated Hospital of Chongqing Medical University, Chongqing 400016, \\ China; ${ }^{3}$ Center for Phenomics of Traditional Chinese Medicine, Southwest Medical University, Luzhou 646000, China; ${ }^{4}$ Laboratory of \\ Cardiovascular Phenomics, Department of Pharmacology, University of Nevada Reno School of Medicine, Reno, NV 89557, USA
}

\begin{abstract}
Lower back pain (LBP) is the most common disease in orthopedic clinics world-wide. A classic Fangji of traditional Chinese medicine, Duhuo Jisheng Decoction (DHJSD), has been proven clinically effective for LBP but its therapeutic mechanisms remain unclear. We hypothesized that DHJSD might relieve LBP through inhibiting the exaggerated proinflammatory cytokines and extracellular matrix (ECM) degradation. Thus, we studied the effects of DHJSD on stromal cell-derived factor-1 (SDF-1)-induced inflammation and ECM degradation in human nucleus pulposus cells (hNPCs). The primary hNPCs were isolated from either degenerated human intervertebral disc (HID) of LBP patients or normal HID of lumbar vertebral fracture patients, and cultured in vitro. The cells were treated with SDF-1 (10 ng/mL) and subsequently with different concentrations (100-500 $\mu \mathrm{g} / \mathrm{mL})$ of DHJSD for $24 \mathrm{~h}$, respectively. We found that application of DHJSD significantly antagonized the SDF-1-induced production of proinflammatory cytokines and reduction of aggrecan and type II collagen in the hNPCs. DHJSD also markedly reduced the SDF-1-induced increase of CXCR4 and p-p65 and inhibited the nuclear translocation of p65 in the hNPCs. DHJSD, CXCR4-siRNA, and NF-kB inhibitor (BAY11-7082) caused the same inhibition of exaggerated proinflammatory cytokines in the SDF-1-treated hNPCs. These results provided compelling evidence that DHJSD may inhibit the generation of proinflammatory mediators and ECM degradation of HID through an orchestrated targeting at multiple molecules in the SDF-1/CXCR4/NF-KB pathway, thus offered novel mechanistic insights into the clinical effectiveness of DHJSD on LBP.
\end{abstract}

Keywords: duhuojisheng decoction; traditional Chinese medicine; SDF-1; NF-kB; degenerative intervertebral disc; lower back pain

Acta Pharmacologica Sinica (2018) 39: 912-922; doi: 10.1038/aps.2018.36; published online 24 May 2018

\section{Introduction}

Lower back pain (LBP) is the most common disease in orthopedic clinics and causes a huge financial burden worldwide ${ }^{[1,2]}$. About $40 \%$ of the patients with LBP are caused by intervertebral disc degeneration ${ }^{[3]}$. But the causative and molecular mechanisms of LBP have not yet been fully elucidated. It has been found that the exaggerated expressions of proinflammatory factors such as tumor necrosis factor- $\alpha$

\footnotetext{
"These authors contributed equally to this work.

*To whom correspondence should be addressed.

E-mail langmanzhishen2008@163.com (Chuan MA); 565409672@qq.com (Zong-chao LIU)

Received 2017-09-01 Accepted 2018-04-23
}

(TNF- $\alpha$ ) and interleukin-1 $\beta$ (IL-1 $\beta$ ) is positively correlated with intervertebral disc degeneration ${ }^{[4]}$. An increase of proinflammatory factors promoted the extracellular matrix (ECM) degradation, which is a key cause of intervertebral disc degeneration ${ }^{[5]}$. Also involved in the inflammation and degradation are a small family of soluble chemically attractive cytokines that regulate the recruitment of peripheral response cells $^{[5,6]}$. One of the most widely studied chemokines is stromal cell-derived factor-1 (SDF-1 $)^{[5,6]}$. The main function of SDF-1 is to guide cell migration and to further stimulate the chemotaxis of inflammatory cells and induce cell surface to produce integrins. Our previous studies have shown that the SDF-1 expression and apoptosis in degeneration of human 
intervertebral disc (HID) tissues were significantly higher than in normal HID tissues ${ }^{[6]}$. It has also been reported that SDF-1 promotes the metabolism of chondrocytes by stimulating the release of matrix metalloproteinase-1 (MMP-1) and -13 (MMP-13) in osteoarthritis ${ }^{[7]}$. SDF-1activates various cells by binding to the G-protein-coupled receptor (GPCR), C-X-C motif chemokine receptor $4(\mathrm{CXCR} 4)^{[7,8]}$. CXCR4 has been recognized as the only receptor for SDF-1 and is the only regulator of SDF-1-mediated biological activity ${ }^{[9]}$. SDF-1a/ CXCR4 promote inflammation of the vessel wall ${ }^{[9,10]}$. The SDF-1a / CXCR4 axis inhibitor AMD3100 can reduce the expression of inflammatory factors in lung tissue and airway inflammation as well as airway hyperresponsiveness ${ }^{[9,11]}$. In recent years, nuclear factor-kappa $\mathrm{B}(\mathrm{NF}-\mathrm{kB})$ has been found to be involved in SDF-1 mediated signaling pathways ${ }^{[12-14]}$. NF-kB is a transcription factor, which has an important effect on the response of the cell to injury, stress, and inflammatory reaction. It has been also reported that $\mathrm{NF}-\mathrm{kB}$ plays a significant role in disc degeneration ${ }^{[15-17]}$.

Current treatments of LBP, either surgical or non-surgical, have been usually ineffective and often limited by side effects ${ }^{[2]}$. However, a widely used classic fangji of traditional Chinese medicine (TCM), duhuojisheng decoction (DHJSD), has been proven very effective clinically in the treatment of LBP for thousands of years ${ }^{[18-37]}$. In TCM, LBP is defined as a part of "feng-han-shi" "Bi Zheng" caused by deficiency in both "Gan" and "Shen" or "Qi" and "Xue". DHJSD is an effective eliminator of "feng-han-shi" through boosting "Gan" and "Shen" functions and therefore can reinforce the bones and tendons and relieve pain of "Bi Zheng" [38]. But the detailed molecular mechanisms for the effectiveness of DHJSD on LBP remain unclear. Although some modern pharmacological studies have demonstrated that DHJSD may have effects of analgesia, immune function regulation, blood vessel dilation, anti-inflammation, and antiplatelet aggregation $[20,22,23,26,39,40]$, the mechanisms of the therapeutic effect of DHJSD on HID degeneration are unknown. In this study we used the unique model of SDF-1-treated human nucleus pulposus cells (hNPCs) to test our hypothesis that DHJSD may retard HID degeneration and relieve the LBP through an orchestrated inhibition of the exaggerated production of multiple proinflammatory cytokines and ECM degradation in HID.

\section{Materials and methods DHJSD aqueous extract preparation}

The origin, medicinal composites, and processing technology of DHJSD were standardized based on marker compounds to achieve quality control according to the Chinese Pharmacopoeia 2015 (Chinese Pharmacopeia Commission: Pharmacopoeia of the People's Republic of China. Chinese Medical Science and Technology Press; Beijing, China, 2010), the same as previously described and published ${ }^{[20,22,23,26,39,40]}$. Briefly, the 15 components of DHJSD, namely $9 \mathrm{~g}$ of Duhuo (Radix Angelicae Pubescentis), $6 \mathrm{~g}$ of Jisheng (Herba Taxilli), Qinjiao (Radix Gentianae Macrophyllae), Fangfeng (Radix Saposhnikoviae), Xixin (Herba Asari), Rougui (Cortex Cinnamomi), Fuling
(Poria Cocos), Chuanxiong (Rhizoma Chuanxiong), Danggui (Radix Angelicae Sinensis), Niuxi (Radix Achyranthis Bidentatae), Dihuang (Radix Rehmanniae Preparata), Baishao (Radix Paeoniae Alba), Duzhong (Cortex Eucommiae Ulmoidis), Renshen (Panax ginseng) and Gancao (Radix Glycyrrhizae) ${ }^{[20,22,23,26,39,40]}$, were mixed and soaked in distilled water, rotary evaporator for rotary steam and the solution was filtered and concentrated ${ }^{[41]}$. After boiled for 30 min twice the DHJSD solution was filtered (filter $0.22 \mu \mathrm{m}$ ) and then dissolved in DMEM/F12 medium containing 15\% fetal bovine serum at a final concentration of $10 \mathrm{mg} / \mathrm{mL}$. The solution was subsequently filtered again through a filter $(0.22 \mu \mathrm{m})$ and stored at $4{ }^{\circ} \mathrm{C}$ as a stock solution before use. In the rest of the experiments, the stock DHJSD solution $(10 \mathrm{mg} / \mathrm{mL})$ was further dissolved into the medium with final corresponding concentrations of $100,200,300,400$ and $500 \mu \mathrm{g} / \mathrm{mL}$ of DHJSD.

\section{Primary hNPC isolation and culture}

The study protocol of using human tissues was approved by the Ethics Committee of Southwest Medical University (Luzhou, China). Written informed consent was obtained from all tissue donors before their surgeries. All degenerative NP tissues were acquired from lumbar spine surgery patients. The relatively normal HID tissues as control were obtained from 5 volunteers 2 females and 3 males aged 20-28 (24.4 \pm 3.3$)$ years old] with lumbar vertebral fracture (LVF) who did not have a documented medical history of LBP. The human IDD tissues were obtained from 15 patients with lumbar disc herniation (LDH) [5 females and 10 males aged 49-72 (58.2 \pm 7.3 ) years old]. The degree of intervertebral disc degradation (IVDD) was assessed according to Pfirrmann classification by preoperative magnetic resonance imaging scans ${ }^{[42]}$. The IVDD group exhibited Pfirrmann grades III$\mathrm{V}$. The human NP tissues were microscopically isolated from IVDD tissues by a scalpel under sterile conditions. Then the tissues were washed twice with PBS and cut into $1 \mathrm{~mm}^{3}$ fragments. The fragments of NP tissues were digested in $0.25 \%$ trypsin solution for $30 \mathrm{~min}$ followed by $0.2 \%$ type II collagenase for $3-4 \mathrm{~h}$ at $37^{\circ} \mathrm{C}$. Tissue debris was removed by passing through a $200-\mu \mathrm{m}$ filter and then the NP cells were resuspended in DMEM/F12 containing 15\% FBS and 1\% penicillin-streptomycin at $37^{\circ} \mathrm{C}$ in a humidified atmosphere containing $5 \% \mathrm{CO}_{2}$. When the cells grew to confluence of $80 \%-90 \%$, they were digested by $0.25 \%$ trypsin solution and subcultured in culture flasks. The third generation of hNPCs was used for all experiments.

\section{Cell viability assay}

Cell viability of hNPCs was determined by the Cell Counting Kit-8 (CCK-8) assay according to the manufacturer's instruction (Dojindo Laboratories, Kumamoto, Japan). Briefly, hNPCs were seeded in a 96-well plate $(100 \mu \mathrm{L} /$ well $)$ at a density of $1 \times 10^{4}$ cells $/ \mathrm{mL}$ and cultured for $24 \mathrm{~h}$. They were subsequently treated with or without $10 \mathrm{ng} / \mathrm{mL}$ SDF-1 (Sigma-Aldrich, St Louis, MO, USA) and various concentrations of DHJSD (100, 200, 300, 400 and $500 \mu \mathrm{g} / \mathrm{mL}$ ) for $24 \mathrm{~h}$, respectively. After 
$24 \mathrm{~h}$ incubation, $10 \mu \mathrm{L}$ of the WST-8 reagent [2-(2-methoxy4-nitrophenyl)-3-(4-nitrophenyl)-5-(2,4-disulfonyl)- $2 \mathrm{H}$ tetrazolium] was added to each well, and the cells were incubated at $37^{\circ} \mathrm{C}$ for $2 \mathrm{~h}$. The absorbance of the wells was measured at $450 \mathrm{~nm}$ by a Bio-Rad 680 microplate reader (BioRad, Hercules, CA, USA). Each experiment was performed in three replicate wells.

\section{Cell treatments}

After the cell viability assay with CCK-8, the hNPCs were treated with control, SDF-1 (10 ng/mL), or DHJSD + SDF-1 for $24 \mathrm{~h}$. Double-stranded small interfering RNA (siRNA) targeting CXCR4 (CXCR4-siRNA) was transfected into the cultured cells using PepMutesiRNA Transfection Reagent (Signa Gen Laboratories, Rockville, MD, USA) according to the manufacturer's protocol (Santa Cruz Biotechnology, Inc, Dallas, TX, USA; cat. no. Sc-35422). After transfection with 50 nmol/L CXCR4-siRNA for $72 \mathrm{~h}$, the cultured cells were treated with or without SDF-1 (10 ng/mL) and DHJSD $(300 \mu \mathrm{g} / \mathrm{mL})$ for $24 \mathrm{~h}$ at $37^{\circ} \mathrm{C}$.

\section{Fluorescence immunocytochemistry}

After each group of cells were treated, cells cultured in 24-well plates were washed by PBS for several times and then were fixed with $4 \%$ paraformaldehyde for $10 \mathrm{~min}$. the cells were blocked with normal goat serum for $1 \mathrm{~h}$, and then were incubated with p65 antibody $(1: 100)$ at $4{ }^{\circ} \mathrm{C}$ overnight. Finally, Nuclear counterstaining was incubated with 4',6-diamidino-2-phenylindole. After washed three times with PBS, the cells were observed through a fluorescence microscope.

\section{Real-time PCR analysis}

The total RNA was extracted from human NP tissues using TRIzol reagent (Invitrogen, USA) according to the manufacturer's instructions. An ultraviolet spectrophotometer (Olympus, Japan) was used to measure the purity and concentration of RNA. Next, $1 \mu \mathrm{g}$ of mRNA was reverse transcribed to cDNA. The cDNA samples were amplified by performing real-time PCR in ABI Prism 7500 (ABI, USA) by using SYBR ${ }^{\circledR}$ Green Real-Time PCR Master Mix (TOYOBO, QPK-201). Relative expression levels of the indicated genes were calculated using $2^{-\Delta \Delta \mathrm{Ct}}$ method. All primers were synthesized by TaKaRa (TaKaRa, China). The Primers for human genes were in Table 1.

\section{ELISA}

After each group of cells were treated for $24 \mathrm{~h}$, the supernatants were collected and centrifuged to remove cell fragments. The levels of TNF- $\alpha$ and IL- $1 \beta$ in the supernatant were measured using an ELISA kit (R\&D Systems, Inc. Minneapolis, MN, USA) and a microplate reader (Omega BioTek, Inc) according to the manufacturer's instructions.

\section{Western blot analysis}

Total protein was extracted using radio-immunoprecipitation
Table 1. Primers for PCR.

\begin{tabular}{ll}
\hline Gene & \multicolumn{1}{c}{ Primer Sequence } \\
\hline GAPDH & Sense: 5'-GCACCGTCAAGGCTGAGAAC-3' \\
& Antisense: 5'-TGGTGAAGACGCCAGTGGA-3' \\
IL-1 $\beta$ & Sense: 5'-GAAATGATGGCTTATTACAGTGGC-3' \\
& Antisense: 5'-GCCACTGTAATAAGCCATCATTTC-3' \\
TNF- $\alpha$ & Sense: 5'-TCATCTACTCCCAGGTCCTCTTCA-3' \\
& Antisense: 5'-TGAAGAGGACCTGGGAGTAGATGA-3' \\
MMP-3 & Sense: 5'-ATTCCATGGAGCCA GGCTTTC-3' \\
& Antisense: 5'-CATTTGGGTCAAACTCCAACTGTG-3' \\
MMP-13 & Sense: 5'-TTGATGATGATGAAACCTGGACAAG-3' \\
& Antisense: 5'-TTGCCGGTGTAGGTGTAGATAGGAA-3'
\end{tabular}

assay (RIPA) lysis buffer containing a mixture of protease inhibitor, and protein concentration was measured by Enhanced BCA Protein Assay Kit (Beyotime, P0010S). Protein samples were mixed with sodium dodecyl sulfate (SDS)-polyacrylamide gel electrophoresis (PAGE) sample loading buffer, boiled for $5 \mathrm{~min}$. After electrophoresed in $6 \%-12 \%$ SDS-PAGE gels, protein samples were transferred to polyvinylidene difluoride membrane (PVDF, 0.45 or $0.22 \mu \mathrm{m}$ ). The membranes were blocked with $5 \%$ nonfat dry milk in trisbuffered saline (TBST) for $1 \mathrm{~h}$ and incubated with primary antibodies rabbit anti-p-p65 and anti-p65 (Cell Signaling Technology, Inc, Beverly, MA, USA), rabbit anti-MMP-3 and anti-MMP-13 (Epitomics), rabbit anti-CXCR4 (Cell Signaling Technology, Inc, Beverly, MA, USA), mouse anti-TNF-a (OriGene, Herford, Germany), mouse anti-IL-1 $\beta$ (OriGene) and mouse anti- $\beta$-actin (Santa Cruz Biotechnology, Inc, Santa Cruz, CA, USA) overnight at $4^{\circ} \mathrm{C}$. After washed three times for $10 \mathrm{~min}$ in TBST, the membranes were incubated in secondary antibody for $2 \mathrm{~h}$. Finally, the membranes were treated with ECL plus reagent (Invitrogen, USA) and the results were analyzed by the accompanied software.

\section{Statistical analysis}

All measurements were performed in triplicate, and results were expressed as mean \pm standard deviation (SD). Statistical analysis was performed by one-way analysis of variance (ANOVA) or Student's t-test using SPSS 19.0 (IBM, Corp, Armonk, NY, USA). $P<0.05$ was considered to indicate a statistically significant difference.

\section{Results}

Basal expression of TNF- $\alpha$, IL-1 $\beta$, MMP-3 and MMP-13 in human NP tissues

The expression levels of TNF- $\alpha$, IL-1 $\beta$, MMP-3 and MMP-13 were significantly increased in the degenerative human NP tissues compared with the normal NP tissues as revealed by PCR (Figure 1A) and Western blot (Figure 1B) analyses. These results suggest that the increase of multiple proinflammatory factors and extracellular matrix (ECM) degradation may play a critical role in the process of human IVDD. 
A

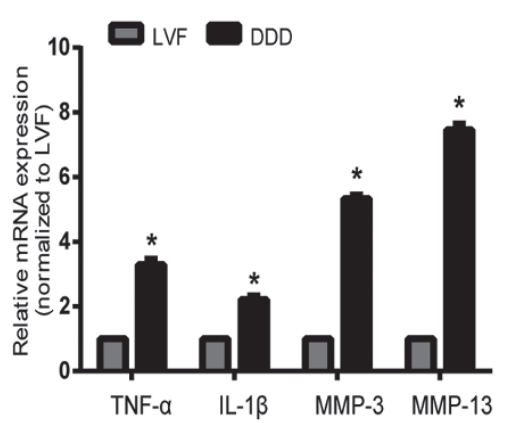

B

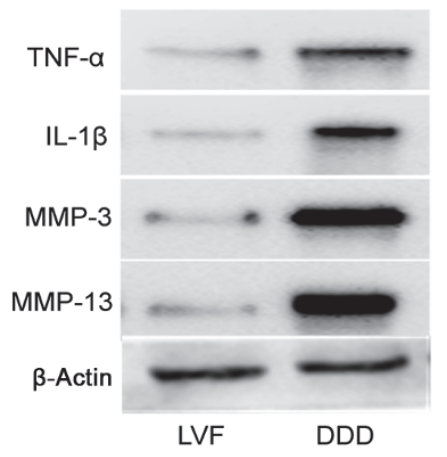

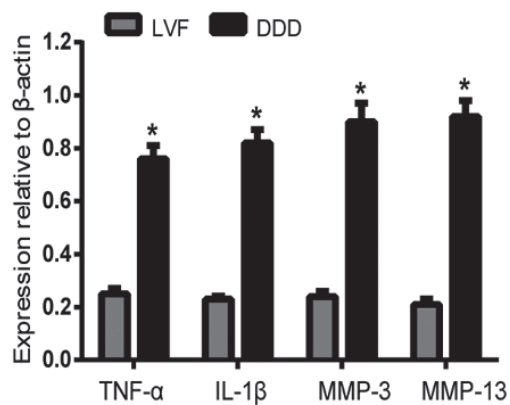

Figure 1. Expression of proinflammatory factors in human nucleus pulposus cells (hNPCs). Real-time PCR (A) and Western blot (B) analyses for TNF- $\alpha$, IL-1 $1 \beta$, MMP-3, and MMP-13 expression in hNPCs from patients with lumbar vertebra fracture (LVF) and disc degenerative disease (DDD). Data represents mean \pm SD, ${ }^{*} P<0.05$ vs LVF group.

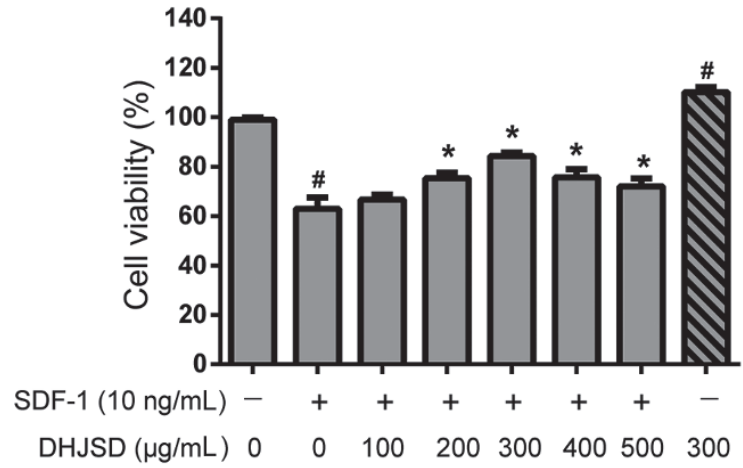

Figure 2. Duhuo Jisheng Decoction (DHJSD) on cell viability of hNPCs in the absence and presence of SDF-1. Cells were treated with SDF-1 (10 $\mathrm{ng} / \mathrm{mL}$ ) and SDF-1 plus various DHJSD concentrations (100, 200, 300, 400 and $500 \mu \mathrm{g} / \mathrm{mL}$ ) as well as $300 \mu \mathrm{g} / \mathrm{mL}$ DHJSD alone (without SDF1) for $24 \mathrm{~h} .{ }^{\#} P<0.05$ compared to untreated group (without SDF-1 or DHJSD); ${ }^{*}<<0.05$ compared with SDF-1 treated group.

\section{Effects of DHJSD on cell viability of SDF-1-treated hNPCs}

To investigate the effects of DHJSD on cell viability, hNPCs were treated with either SDF-1 (10 ng/mL) or SDF-1 plus various concentrations of DHJSD for $24 \mathrm{~h}$, followed by analysis with the CCK-8 viability assay. As shown in Figure 2, SDF-1 significantly reduced the viability of hNPCs to $62.98 \% \pm 4.48 \%$ $(P<0.05$ compared to the group of untreated cells) while DHJSD $(300 \mu \mathrm{g} / \mathrm{mL})$ alone promoted the cell viability of hNPCs to $110.23 \% \pm 1.89 \%(P<0.05$ compared to the group of untreated cells). The addition of various concentrations (100, 200, 300, 400 and $500 \mu \mathrm{g} / \mathrm{mL}$ ) of DHJSD increased the cell viability of SDF-1-induced hNPCs. In the presence of SDF1. the group of $300 \mu \mathrm{g} / \mathrm{mL}$ DHJSD had the highest viability of hNPCs among all concentrations of DHJSD, suggesting 300 $\mu \mathrm{g} / \mathrm{mL}$ DHJSD may produce the maximal effect of enhancing the viability of SDF-1-induced hNPCs. Therefore, we decided to use $300 \mu \mathrm{g} / \mathrm{mL}$ of DHJSD in the rest of experiments to study the effects of DHJSD on the $10 \mathrm{ng} / \mathrm{mL}$ of SDF-1 induced reduction in cell viability of hNPCs.

Effects of DHJSD on the generation of TNF- $\alpha$ and IL-1 $1 \beta$ in hNPCs As shown in Figure 3, SDF-1 significantly increased the mRNA (Figure 3A) and protein (Figure 3B) levels of TNF- $\alpha$ and IL-1 $\beta$ compared to that in the control groups. However, this SDF-1 induced increase in the expression of TNF- $\alpha$ and IL- $1 \beta$ mRNA and protein were markedly antagonized by DHJSD $(P<0.05$ SDF-1+DHJSD vs SDF-1).

\section{Effects of DHJSD on SDF-1 induced hNPCs matrix degradation}

To determine the effect of DHJSD on human IVD degeneration, the mRNA and protein levels of various matrix-degrading enzymes were examined by real-time PCR (Figure 4A) and Western blot (Figure 4B). SDF-1 treatment significantly increased the mRNA and protein levels of multiple MMPs (MMP-3 and MMP-13), but decreased the mRNA and protein levels of aggrecan and collagen II (protein:aggrecan, $P<0.01$; collagen II, $P<0.01$ vs control group). However, DHJSD treatment markedly decreased the mRNA and protein levels of multiple MMPs (MMP-3 and MMP-13) and increased the mRNA and protein levels of aggrecan and collagen II (protein: aggrecan, $P=0.03$; collagen II, $P<0.01$ vs SDF- 1 group), indicating that the SDF-1-induced hNPC matrix degradation could be significantly inhibited by DHJSD.

\section{Effects of DHJSD on the proinflammatory factors generation and} matrix degradation of HID

To understand the mechanisms for the DHJSD inhibition of the SDF-1-induced inflammation and degeneration of hNPCs we further studied the effects of DHJSD on SDF-1-induced proinflammatory factors and matrix degradation. As shown in Figure 5A, the protein levels of CXCR4 and p-p65 were markedly increased in the SDF-1 treated hNPCs group when compared with the control group (CXCR4, P<0.01; p-p65, $P=0.02$ ). However, DHJSD markedly reduced the levels of 
A

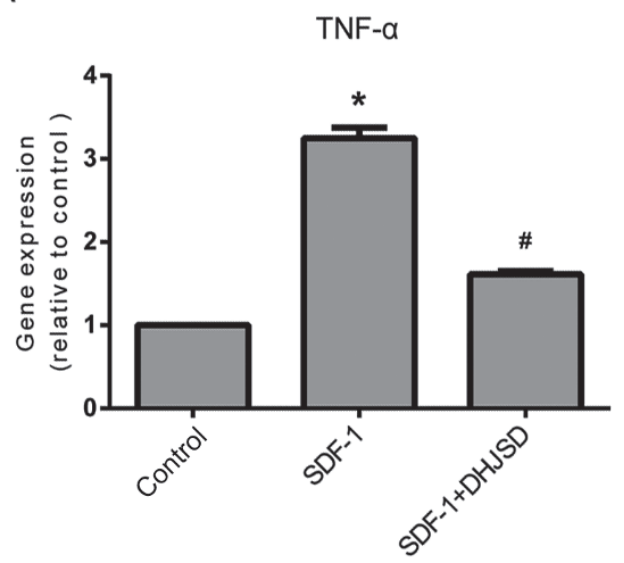

B

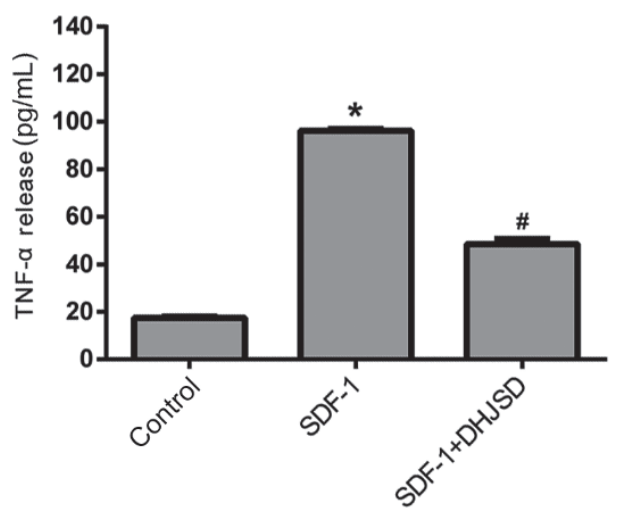

IL-1 $\beta$
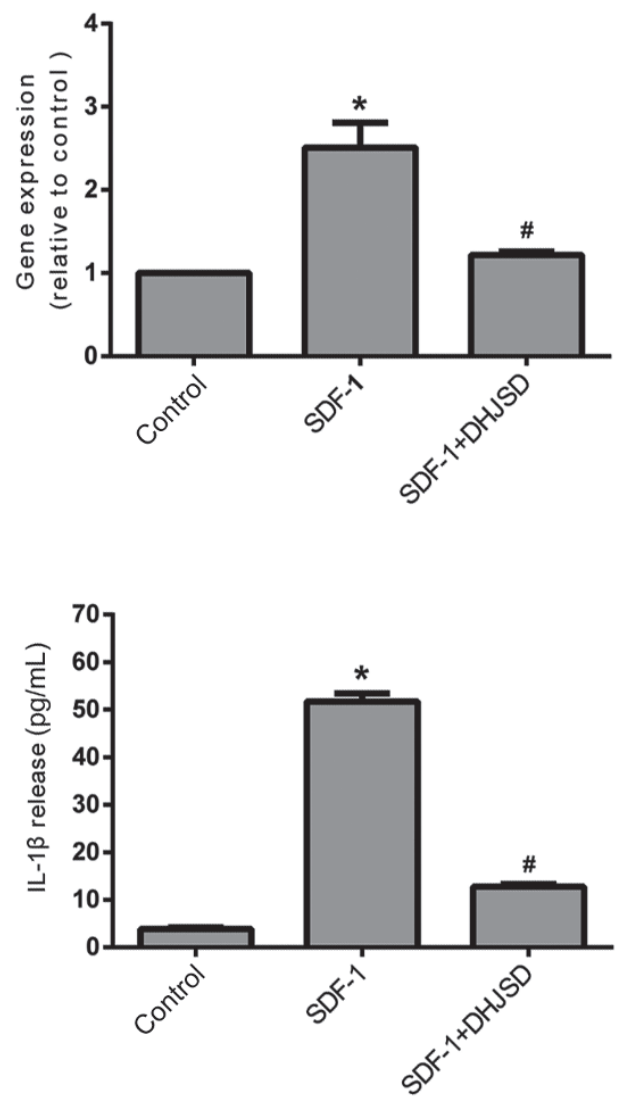

Figure 3. DHJSD on SDF-1 induced expression of TNF- $\alpha$ and IL-1 $\beta$ in human nucleus pulposus cells (hNPCs). (A) The mRNA expressions of TNF- $\alpha$ and $\mathrm{IL}-1 \beta$ were determined by real-time PCR. (B) The secretion of TNF- $\alpha$ and IL-1 $\beta$ from hNPCs into the culture medium were determined by ELISA. ${ }^{*} P<0.05$ compared with control group. ${ }^{\#} P<0.05$ compared with SDF-1 group.

CXCR4 and p-p65 in the SDF-1 treated hNPCs group (CXCR4, $P<0.01$; p-p65, $P=0.02)$. Interestingly, DHJSD caused the same down regulation of TNF- $\alpha$, IL-1 $\beta$, MMP-3 and MMP-13 expression as the CXCR4-siRNA or NF-KB inhibitor (BAY117082) in the SDF-1-treated hNPCs (Figure 5). Furthermore, as shown in Figure 6, DHJSD treatment or down regulation of the CXCR4 expression by CXCR4-siRNA transfection all inhibited the nuclear translocation of p65 in the SDF-1 treated hNPCs. These results strongly suggest that DHJSD may inhibit the generation of human intervertebral disc proinflammatory factors and ECM degradation via targeting at multiple molecules in the SDF-1/CXCR4/NF-kB pathway in a well-orchestrated and integrated fashion.

\section{Discussion}

The major findings in the current study include the pharmacological anti-inflammation and anti-degeneration effects of DHJSD on IVDD through the inhibition of SDF-1induced proinflammatory factors and matrix degradation via targeting multiple molecules in the SDF-1/CXCR4/ NF-kB pathway in hNPCs. It provides novel insights into the molecular mechanisms of DHJSD on human disc degeneration and has significant impact on the validation of clinical application of DHJSD, a classic TCM fangji, for the treatment of LBP caused by human IVDD.

The exaggerated inflammation with the increase of proinflammatory factors in intervertebral disc cells and ECM degradation are considered the major pathologic processes that cause disc degeneration ${ }^{[43]}$. Therefore, inhibition of the proinflammatory factors and ECM degradation in the intervertebral disc may be a very effective mechanism to retard the disc degeneration (Figure 2). IVDD is characterized by an increase in the expression levels of proinflammatory cytokines, including IL-1 $\beta$, which induce ECM degradation, chemokine production and changes in cell phenotype $\mathrm{e}^{[43,44]}$. The release of chemokines promotes the infiltration and activation of immune cells, which amplifies the inflammatory cascade. SDF-1 is highly expressed in inflamed tissues, where it attracts activated $\mathrm{CXCR}^{+} \mathrm{T}$ cells, thus enhancing local inflammatory responses ${ }^{[45]}$. We have previously reported that SDF-1/CXCR4 axis induces apoptosis of degenerative human nucleus pulposus cells via the NF-KB pathway ${ }^{[6]}$. In this study, we further found 
A

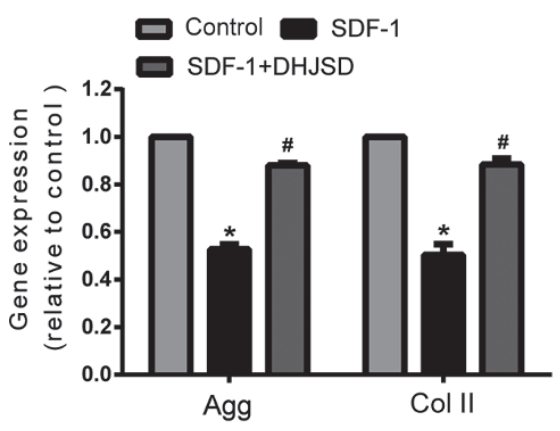

B

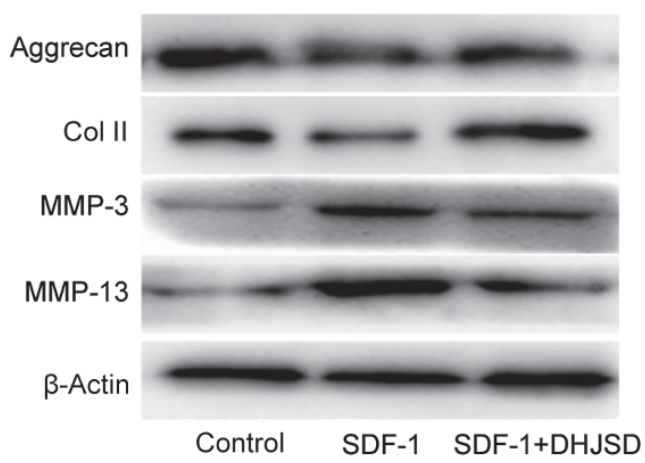

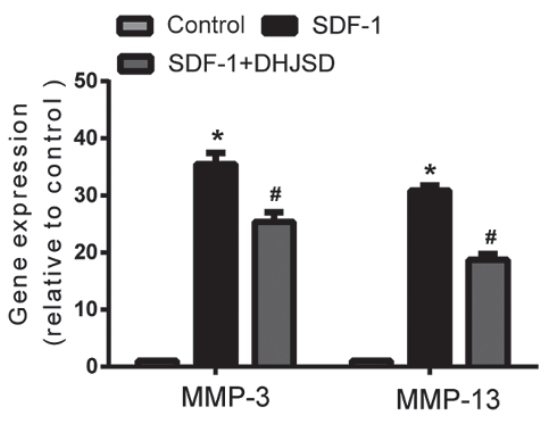

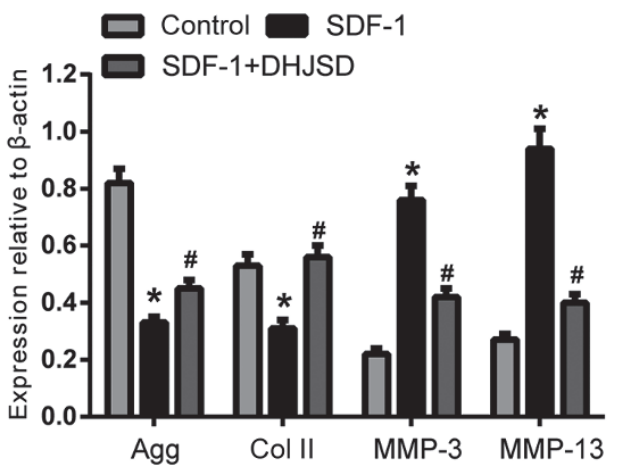

Figure 4. DHJSD on SDF-1-induced matrix degradation. (A) The mRNA expressions of aggrecan, collagen II, MMP-3 and MMP-13 were measured by real-time PCR analysis. (B) The protein expressions of aggrecan, collagen II, MMP-3 and MMP-13 were measured by Western blot analysis. ${ }^{*} P<0.05$ when compared with control group. ${ }^{\#} P<0.05$ when compared with SDF-1 group.

that proinflammatory cytokines and ECM degradation were markedly upregulated in SDF-1-induced human NPCs. Anti-inflammatory strategy with agents or gene therapy has been proven to be effective for delaying disc degeneration in vitro studies, but its clinical efficacy remains to be further investigated $^{[46,47]}$. In this study, we provided compelling evidence that the treatment of IDD with DHJSD significantly reduced the generation of proinflammatory factors and ECM degradation. A screening method was used in the present study to measure SDF-1-induced human NPCs viability by CCK-8 analysis. We found that the minimum dose for DHJSD to reverse SDF-1-induced decrease in NPCs viability was 300 $\mu \mathrm{g} / \mathrm{mL}$. DHJSD significantly reversed the SDF-1-induced upregulation of proinflammatory factors and key molecules in ECM degradation, including TNF- $\alpha$, IL-1 $\beta$, aggrecan, collagen II, MMP-3 and MMP-13, in human NPCs.

The SDF-1/CXCR4 axis has been previously associated with the pathogenesis of chronic inflammatory diseases, including osteoarthritis ${ }^{[6]}$ and rheumatoid arthritis ${ }^{[48]}$. Our previous studies have demonstrated that the SDF-1/CXCR4 axis is involved in the IVDD process ${ }^{[4]}$. The results from the current study provided novel evidence that the SDF-1/ CXCR4 axis also regulates many inflammatory responses and ECM degradation in IVDD. The NF-kB pathways have been reported to play important roles in the regulation of inflammatory response and ECM degradation ${ }^{[50,51]}$. Here, for the first time in literature, we found that DHJSD treatment had same inhibitory effects as the CXCR4 knock down by CXCR4-siRNA and NF-kB inhibition by BAY11-7082 on the SDF-1-induced expression of TNF- $\alpha$, IL-1 $\beta$, MMP-3, MMP13, CXCR4 and p-p65, and simultaneously suppressed p65 nucleus translocation. These results strongly suggest that DHJSD exerts its anti-inflammation and anti-degeneration effects through an orchestrated and integrated targeting at multiple key molecules in the SDF-1/CXCR4/NF-кB pathway. This may explain the unique effectiveness of the TCM fangji DHJSD and its advantages over the conventional non-steroid anti-inflammatory drugs (NSAIDs) in the treatment of LBP.

It is important to understand that due to the uniqueness of the composition theory of effective TCM fangji the study of TCM pharmacology needs a revolutionary paradigm shift in terms of rationales and approaches. It is now the time to consider a new knowledge network for deep understanding of an integrated and orchestrated combination of multi-targets for precision medicine, instead of an accumulation of single molecular target. In TCM, LBP is diagnosed as a "feng-hanshi"-caused "Bi Zheng", which usually attacks patients with dual deficiencies in both "Gan" and "Shen" ${ }^{[52]}$. Here, we 
A
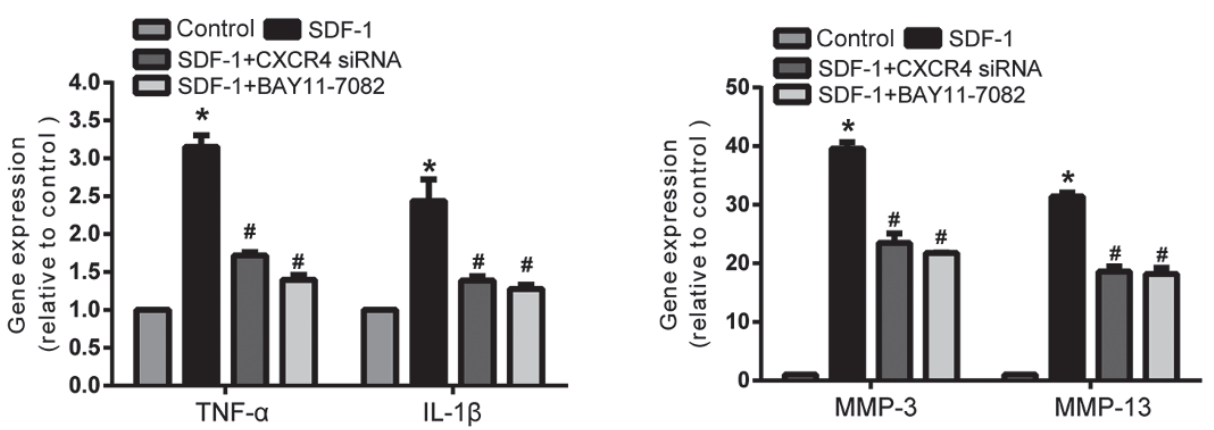

B
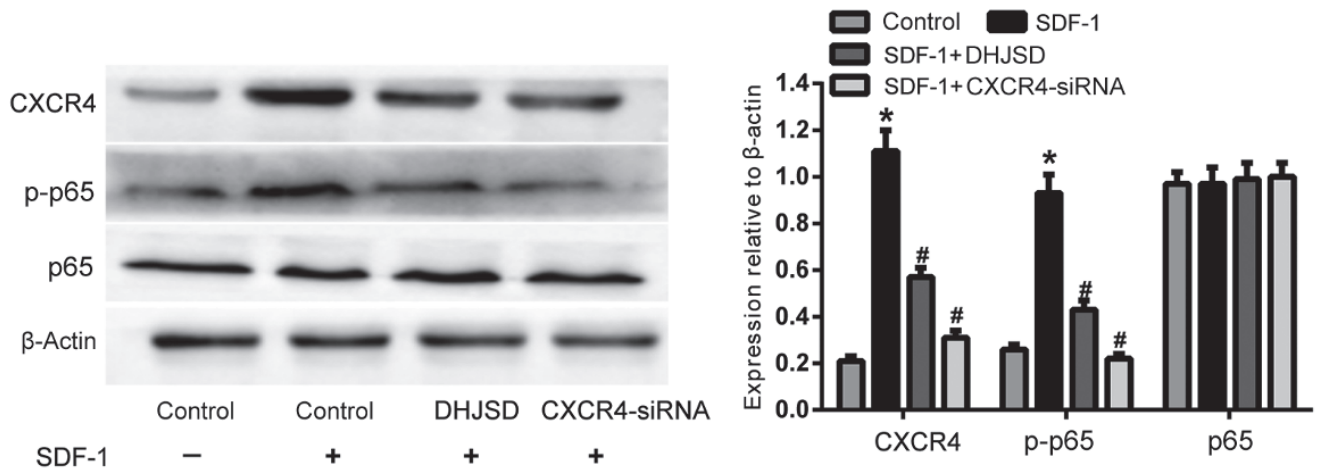

Figure 5. Effects of DHJSD on human intervertebral disc proinflammatory factors and ECM degradation through the SDF-1/CXCR4/NF-KB pathway. (A) Effects of NF-KB inhibitor, BAY11-7082, and CXCR4 siRNA in the presence of SDF-1 on the expression of TNF- $\alpha$, IL-1 13, MMP-3 and MMP-13 measured by real-time PCR analysis. (B) Western blot analysis of DHJSD and CXCR4-siRNA on the protein expression of CXCR4, p-p65 and p65 in the hNPCs after treated with SDF-1. Data are presented as the mean \pm standard deviation from three independent experiments. ${ }^{*} P<0.05$ vs the control group. ${ }^{\#} P<0.05$ vs SDF- 1 group.

prefer to use the original TCM definition of "Gan" and "Shen" instead of the commonly used but confusing terms of "liver" and "kidney", respectively, because they are totally different in terms of organs and functions. Similarly, the TCM terms of "Qi" and "Xue" are not the same as "air" and "blood".

While the TCM terms of "Gan", "Shen", "Qi", and "Xue" for these particular etiological problems are complicated to be interpreted in Western medicine terms, a corresponding TCM fangji, DHJSD, was specifically developed with a unique integrated combination strategy to orchestrate the targeting at the primary and secondary causatives according to the principles of "Jun, Chen, Zuo, and Shi" ${ }^{131]}$. As shown in Figure 7, DHJSD has an overall effect to eliminate "fenghan-shi", respectively, and thus to relieve pain of "Bi Zheng" through promoting "Gan" and "Shen" function and therefore strengthen the bones and tendons ${ }^{[30]}$. It also improves circulation and remove stasis of "Qi" and "Xue", reduces tissue swelling and relieves pain ${ }^{[20,22,23,26,32,39,40]}$. Some pharmacological studies have demonstrated that DHJSD may have effects of analgesia, immune function regulation, blood vessel dilation, anti-inflammation, and antiplatelet aggregation ${ }^{[20,22,23,26,32,39,40]}$. In a study of the aging-related osteoarthritis, another common bone degenerative disease, Chen et al found that DHJSD may inhibit VEGF and
HIF-1a to retard cartilage degradation in a rabbit model of osteoarthritis ${ }^{[53]}$. How to translate these findings into the mechanisms of DHJSD on the degeneration of human intervertebral disc remains elusive. DHJSD derives its name from two of the principal ingredients, duhuo (contains osthole) and jisheng as "Jun" (or chief) components to target the major etiological problems, which is characterized by the strong effect on chronic "Bi" pain and numbness by eliminating "feng-han-shi", in the lower part of the "three jiaos" ${ }^{[21]}$. Its pharmaceutical actions are antirheumatic, anti-inflammatory, and analgesic (pain relieving) and thus it is specific for rheumatic pain that is sensitive to cold, especially if it occurs in the lower body. Previous quality control studies found that osthole exhibited anti-inflammation ${ }^{[54,55]}$ and anti-bone resorption effect ${ }^{[56]}$ and stimulated osteoblast differentiation and bone formation by activation of beta-catenin-BMP signaling ${ }^{[57]}$. Jisheng has dual functions: it strengthens the "Gan" and "Shen" while subsequently nourishing the bones and tendons and relieves the pain of arthritis. Other ingredients in the formula include the basic formulas to tonify both "Qi" and "Xue", which assist the remedy in returning strength, vitality, and strong immune function, making it especially useful for long-term use by elderly or weakened patients. The "chen" components all facilitate the effect 


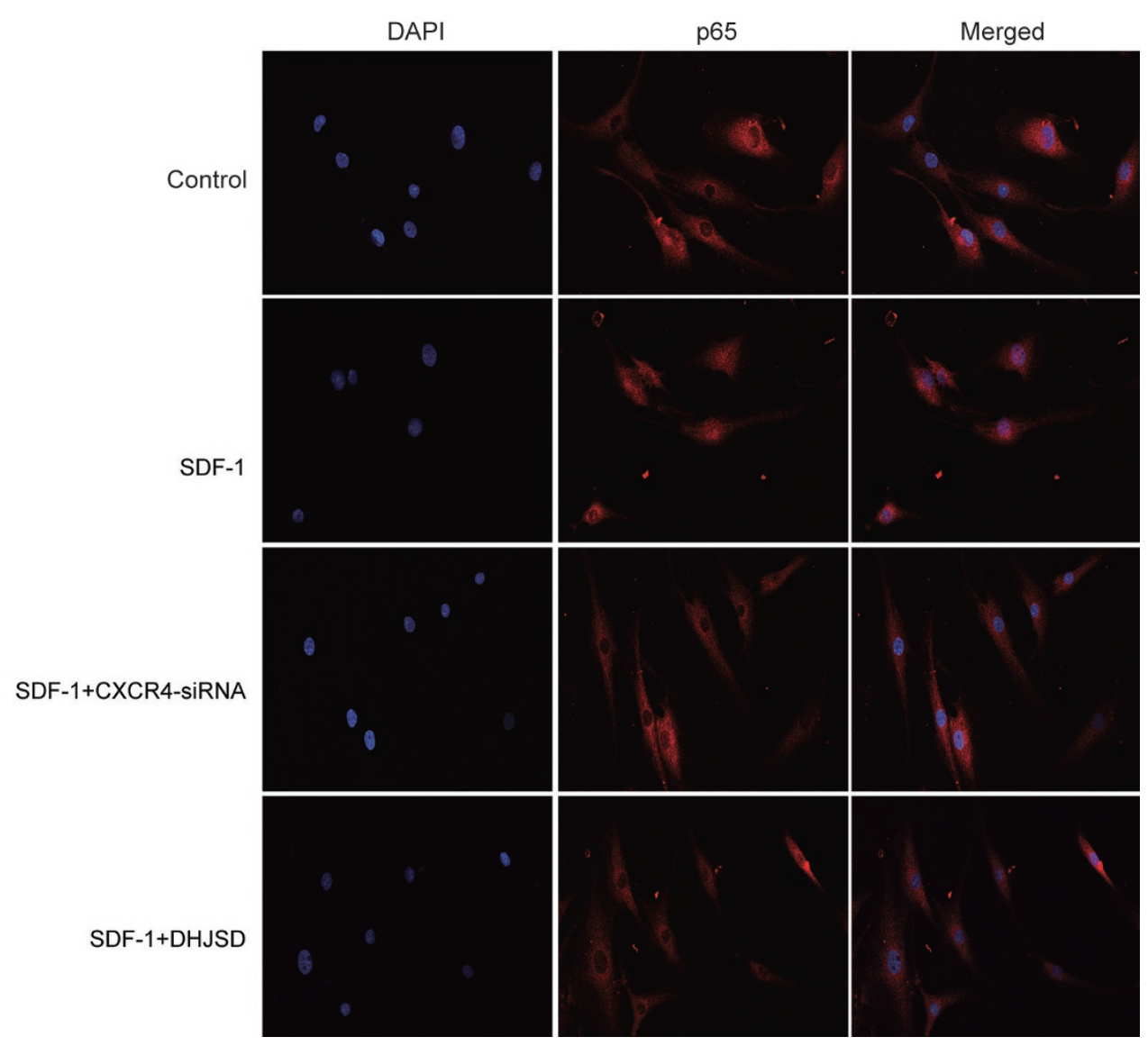

Figure 6. Effects of DHJSD on the nuclear translocation of p65 in the SDF-1 treated hNPCs. Fluorescence immunocytochemistry was used to detect nuclear translocation of $\mathrm{p} 65$.

of du-huo on "feng-han-shi" by targeting the secondary etiological problems, include fang-feng for systematic "fenghan-shi", xi-xin for "feng-han-shi" in the "Shen", rougui for improving circulation and eliminating "han", and finally qinjiao for "feng-han-shi" in the joints. A very recent report found that qin-jiao had a significant protective effect on the rat model of osteoarthritis and the mechanisms may involve the inhibition of the Rho/NF-kB and TGF- $\beta$ /smad-3 pathways ${ }^{[58]}$. The "Zuo" components include duzhong, niuxi, were used to promote function of "Gan" and "Shen" and strengthen the tendons and bones. Danggui, chuanxiong, dihuang, and baishao were used also as "Zuo" components to nourish and modulate function of "Xue". Renshen, fuling and gancao were used to promote function of " $\mathrm{Pi}$ " and "Qi". The integrated function of the DHJSD is therefore an effective "feng-han-shi" eliminator and LBP reliever. Indeed, previous quality control study of DHJSD suggested that this fangji comprises mainly osthole, gentiopicroside, loganic acid, and paeoniflorin $^{[59,60]}$. While osthole had anti-inflammation ${ }^{[54,55]}$ and anti-bone resorption effect ${ }^{[56]}$ and could stimulate osteoblast differentiation and bone formation by activation of beta-catenin-BMP signaling ${ }^{[57]}$, gentiopicroside has analgesic effects in the mice ${ }^{[61]}$. Loganic acid decreased proinflammatory cytokines in hypercholesterolemic rabbits ${ }^{[62,63]}$. Paeoniflorin is able to suppress inflammation in experimental arthritis by inhibiting abnormal proliferation of lymphocytes and synoviocytes and the production of proinflammatory cytokines and chemokines, nitric oxide, vascular endothelial growth factor (VEGF), and GM-CSF by synoviocytes ${ }^{[19,64-66]}$. Ferulic acid, isolated from chuanxiong and danggui ${ }^{[67]}$ has antioxidative and anti-inflammatory effects ${ }^{[67,68]}$. All the above study indicates that DHJSD has anti-inflammatory actions, consistent with the result in current study that DHJSD has an integrated anti-inflammatory effect and significant protective effect against the ECM degeneration of the SDF-1-treated hNPCs. While it warrants to further dissect the detailed mechanism of the synchronization of this classic TCM fangji in the treatment of LBP, we provided the very first compelling evidence at the molecular level that DHJSD may inhibit the generation of proinflammatory factors and ECM degradation of human intervertebral disc via targeting multiple key molecules in the SDF-1/CXCR4/NF-kB pathway in an orchestrated manner (Figure 7). These studies open a new field of research on the detailed mechanisms of TCM fangji of DHJSD through a Fangjiomics approach ${ }^{[69,70]}$.

In conclusion, DHJSD inhibits the generation of SDF-1induced human intervertebral disc proinflammatory factors and ECM degradation via the SDF-1/CXCR4/NF-kB pathway. 


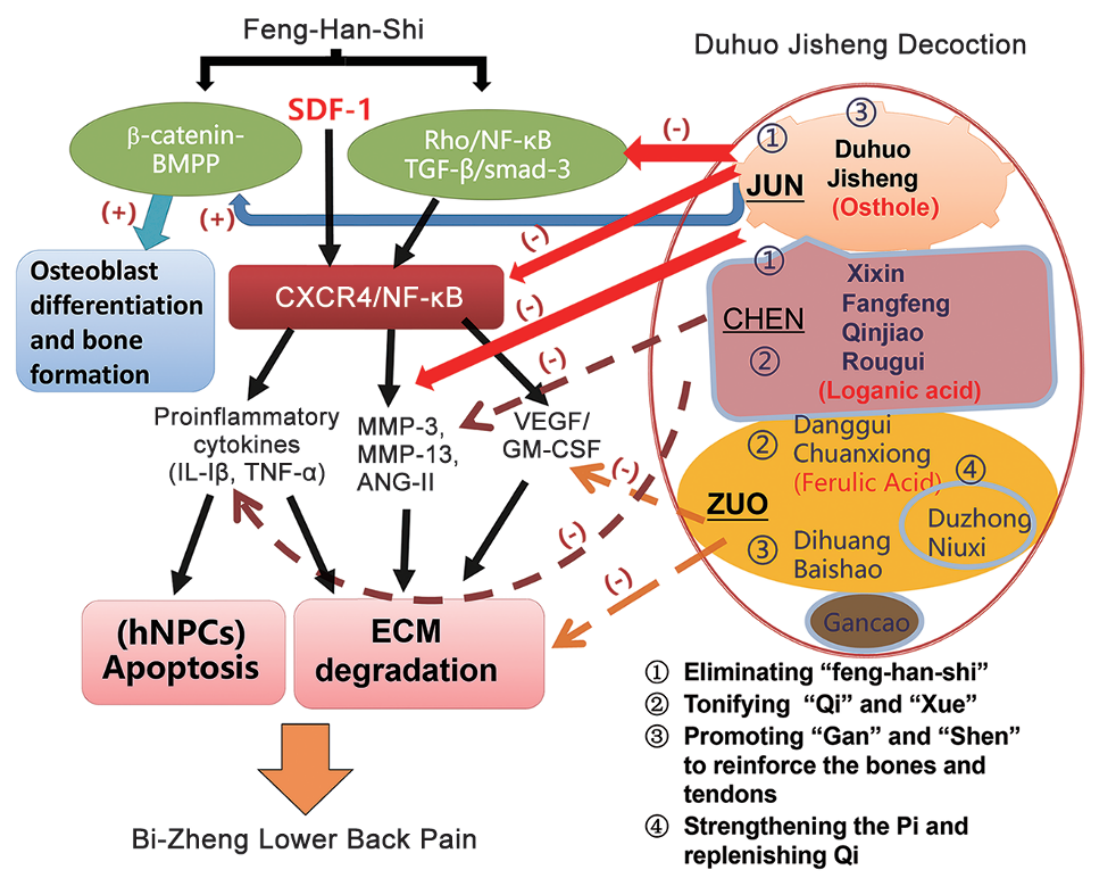

Figure 7. Schematic summary of molecular mechanisms for duhuo jisheng decoction (DHJSD) on the lower back pain (LBP) caused by Bi Zheng. In TCM, "Bi Zheng" LBP is caused by Feng-Han-Shi due to double deficiencies in "Gan" and "Shen" or in "Qi" and "Xue" [54]. DHJSD was specifically developed to orchestrate the therapeutic actions to target at the primary (Jun) and secondary (Chen and Zuo) causatives [31] and has an overall effect to eliminate "feng-han-shi" and thus relieves pain of "Bi Zheng" through promoting "Gan" and "Shen" function and reinforcing the bones and tendons [30]. It also improves circulation and removes stasis of "Qi" and "Xue", reduces tissue swelling, and relieves pain [20, 22, 23, 26, 32, 39, 40]. Duhuo (contains osthole) and jisheng target the major etiological problems as "Jun" (or chief) components by eliminating "feng-han-shi"[21]. Osthole exhibited antiinflammation [56, 57] and anti-bone resorption effect [58] and stimulated osteoblast differentiation and bone formation by activation of beta-cateninBMP signaling (indicated by the blue arrows) [59]. Jisheng has dual functions: it strengthens the "Gan" and "Shen" while subsequently nourishing the bones and tendons and relieves the pain of arthritis. DHJSD may inhibit VEGF and HIF- $1 \alpha$ to retard cartilage degradation [55]. The "chen" components all facilitate the effect of du-huo on "feng-han-shi" by targeting at the secondary etiological problems through the inhibition of the Rho/NF-kB and TGF- $\beta$ / smad-3 pathways[60]. The "Zuo" components include duzhong, niuxi, were used to promote function of "Gan" and "Shen" and strengthen the tendons and bones, danggui, chuanxiong, dihuang, and baishao to nourish and modulate function of "Xue"; renshen, fuling and gancao to promote function of "Pi" and "Qi". Therefore, the integrated function of the DHJSD is an effective "feng-han-shi" eliminator and LBP reliever and has anti-inflammation [56, 57] and anti-bone resorption effect [58] and can stimulate osteoblast differentiation and bone formation [59] and has an integrated anti-inflammatory effect and significant protective effect against the ECM degeneration of the SDF-1-treated hNPCs. ECM: extracellular matrix; hNPCs: human nucleus pulposus cells; VEGF: vascular endothelial growth factor; GM-CSF: granulocyte-macrophage colony-stimulating factor; CXCR4: C-X-C motif chemokine

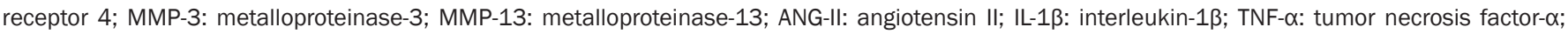
SDF-1: stromal cell-derived factor-1 (+) stimulation, (-) inhibition.

DHJSD may be used as an effective therapeutic agent for the treatment of IVDD-induced LBP.

\section{Acknowledgements}

This work was supported from Southwest Medical University (2014QN-057).

\section{Author contribution}

Chuan MA designed the experiments and overviewed the study; Zong-chao LIU and Zhen-long WANG conducted the experiments and wrote the manuscript; Zhi-jiang FU, Chen-yi HUANG, Yong LIU, Zhang-chao WEI, Shi-gui LIU, and Jie-liang SHEN performed part of the experiments; Zongchao LIU evaluated the final results; Dayue Darrel DUAN participated in writing and finalizing the manuscript.

\section{References}

1 McBeth J, Jones K. Epidemiology of chronic musculoskeletal pain. Best Pract Res Clin Rheumatol 2007; 21: 403-25.

2 Castillo ER, Lieberman DE. Lower back pain. Evol Med Public Health 2015; 2015: 2-3.

3 Sharma A, Sharma SK, Wanchu A, Kumar M, Singh S, Bambery P. Lower-back pain, intervertebral-disc calcification and scleral pigmentation. Med J Aust 2006; 184: 643.

4 Wang J, Markova D, Anderson DG, Zheng Z, Shapiro IM, Risbud MV. TNF-alpha and IL-1beta promote a disintegrin-like and metalloprotease with thrombospondin type I motif-5-mediated aggrecan degradation through syndecan-4 in intervertebral disc. J Biol Chem 2011; 286 : 39738-49.

5 Markova DZ, Kepler CK, Addya S, Murray HB, Vaccaro AR, Shapiro IM et al. An organ culture system to model early degenerative changes of the intervertebral disc II: profiling global gene expression changes. Arthritis Res Ther 2013; 15: R121. 
6 Liu Z, Ma C, Shen J, Wang D, Hao J, Hu Z. SDF1/CXCR4 axis induces apoptosis of human degenerative nucleus pulposus cells via the NFkappaB pathway. Mol Med Rep 2016; 14: 783-9.

7 Pulsatelli L, Dolzani P, Piacentini A, Silvestri T, Ruggeri R, Gualtieri G et al. Chemokine production by human chondrocytes. J Rheumatol 1999; 26: 1992-2001.

8 Ratajczak MZ, Majka M, Kucia M, Drukala J, Pietrzkowski Z, Peiper $\mathrm{S}$, et al. Expression of functional CXCR4 by muscle satellite cells and secretion of SDF-1 by muscle-derived fibroblasts is associated with the presence of both muscle progenitors in bone marrow and hematopoietic stem/progenitor cells in muscles. Stem Cell 2003; 21 : 363-71.

9 Massberg S, Konrad I, Schurzinger K, Lorenz M, Schneider S, Zohlnhoefer $D$, et al. Platelets secrete stromal cell-derived factor 1alpha and recruit bone marrow-derived progenitor cells to arterial thrombi in vivo. J Exp Med 2006; 203: 1221-33.

10 Gear AR, Camerini D. Platelet chemokines and chemokine receptors: linking hemostasis, inflammation, and host defense. Microcirculation 2003; 10: 335-50.

11 Lukacs NW, Berlin A, Schols D, Skerlj RT, Bridger GJ. AMD3100, a CXCR4 antagonist, attenuates allergic lung inflammation and airway hyperreactivity. Am J Pathol 2002; 160: 1353-60.

12 Hsieh YY, Shen CH, Huang WS, Chin CC, Kuo YH, Hsieh MC, et al. Resistin-induced stromal cell-derived factor-1 expression through Toll-like receptor 4 and activation of p38 MAPK/ NFkappaB signaling pathway in gastric cancer cells. J Biomed Sci 2014; 21: 59.

13 Tang $\mathrm{CH}$, Chuang JY, Fong YC, Maa MC, Way TD, Hung CH. Bonederived SDF-1 stimulates IL-6 release via CXCR4, ERK and NF-kappaB pathways and promotes osteoclastogenesis in human oral cancer cells. Carcinogenesis 2008; 29: 1483-92.

14 Tang $\mathrm{CH}$, Tan TW, Fu WM, Yang RS. Involvement of matrix metalloproteinase-9 in stromal cell-derived factor-1/CXCR4 pathway of lung cancer metastasis. Carcinogenesis 2008; 29: 35-43.

15 Zhongyi S, Sai Z, Chao L, Jiwei T. Effects of nuclear factor kappa B signaling pathway in human intervertebral disc degeneration. Spine (Phila Pa 1976 ) 2015; 40: 224-32.

16 Sun Z, Yin Z, Liu C, Tian J. The changes in the expression of NF-KB in a degenerative human intervertebral disc model. Cell Biochem Biophys 2015; 72: 115-22.

17 Xu K, Chen W, Wang X, Peng Y, Liang A, Huang D, et al. Autophagy attenuates the catabolic effect during inflammatory conditions in nucleus pulposus cells, as sustained by NF-kappaB and JNK inhibition. Int J Mol Med 2015; 36: 661-8.

18 Lyu S, Ji B, Gao W, Chen X, Xie X, Zhou J. Effects of Angelicae Pubescentis and Loranthi Decotion on repairing knee joint cartilages in rats. J Orthop Surg Res 2017; 12: 189.

19 Zhang W, Wang S, Zhang R, Zhang Y, Li X, Lin Y, et al. Evidence of Chinese herbal medicine Duhuo Jisheng decoction for knee osteoarthritis: a systematic review of randomised clinical trials. BMJ Open 2016; 6: e008973.

20 Liu F, Weng X, Lin P, Zheng C, Xu H, Liu X, et al. Duhuo Jisheng decoction inhibits endoplasmic reticulum stress in chondrocytes induced by tunicamycin through the downregulation of miR-34a. Int J Mol Med 2015; 36: 1311-8.

21 Shan F, Yuan Y, Hao JD, Huang LQ. Herbal textual research on origin and development of traditional Chinese medicine "duhuo" and “qianghuo". Zhongguo Zhong Yao Za Zhi 2014; 39: 3399-403.

22 Liu F, Liu G, Liang W, Ye H, Weng X, Lin P, et al. Duhuo Jisheng decoction treatment inhibits the sodium nitroprussiateinduced apoptosis of chondrocytes through the mitochondrialdependent signaling pathway. Int J Mol Med 2014; 34: 1573-80.
23 Wu G, Fan H, Huang Y, Zheng C, Ye J, Liu X. Duhuo Jisheng Decoctioncontaining serum promotes proliferation of interleukin1betainduced chondrocytes through the p16 cyclin D1/CDK4Rb pathway. Mol Med Rep 2014; 10: 2525-34.

24 Chen JS, Li XH, Li HT, Weng XP, Xu HF, Ye HZ, et al. Effect of water extracts from duhuo jisheng decoction on expression of chondrocyte $\mathrm{G}_{1}$ phase regulator mRNA. Zhongguo Zhong Yao Za Zhi 2013; 38: 3949-52.

25 Lai JN, Tang JL, Wang JD. Observational studies on evaluating the safety and adverse effects of traditional chinese medicine. Evid Based Complement Alternat Med 2013; 2013: 697893.

26 Wu G, Chen W, Fan H, Zheng C, Chu J, Lin R, et al. Duhuo Jisheng Decoction promotes chondrocyte proliferation through accelerated $\mathrm{G}_{1} / \mathrm{S}$ transition in osteoarthritis. Int J Mol Med 2013; 32: 1001-10.

27 Zheng CS, Xu XJ, Ye HZ, Wu GW, Li XH, Huang SP, et al. Computational approaches for exploring the potential synergy and polypharmacology of Duhuo Jisheng Decoction in the therapy of osteoarthritis. Mol Med Rep 2013; 7: 1812-8.

28 Zhao J, Zha Q, Jiang M, Cao H, Lu A. Expert consensus on the treatment of rheumatoid arthritis with Chinese patent medicines. J Altern Complement Med 2013; 19: 111-8.

29 Hsieh SC, Lai JN, Chen PC, Chen CC, Chen HJ, Wang JD. Is Duhuo Jisheng Tang containing Xixin safe? A four-week safety study. Chin Med 2010; 5: 6.

30 Wu YJ, Luo SF, Yang SH, Chen JY, Yu KH, See LC. Vascular response of Raynaud's phenomenon to nifedipine or herbal medication (duhuotisheng tang with danggui-sini tang): a preliminary study. Chang Gung Med J 2008; 31: 492-502.

31 Xie XX, Lei QH. Observation on therapeutic effect of the spreading moxibustion on rheumatoid arthritis. Zhongguo Zhen Jiu 2008; 28: 730-2.

32 Lai JN, Chen HJ, Chen CC, Lin JH, Hwang JS, Wang JD. Duhuo jisheng tang for treating osteoarthritis of the knee: a prospective clinical observation. Chin Med 2007; 2: 4.

33 Teekachunhatean S, Kunanusorn P, Rojanasthien N, Sananpanich K, Pojchamarnwiputh S, Lhieochaiphunt S, et al. Chinese herbal recipe versus diclofenac in symptomatic treatment of osteoarthritis of the knee: a randomized controlled trial [ISRCTN70292892]. BMC Complement Altern Med 2004; 4: 19.

34 Guo LQ, Taniguchi M, Chen QY, Baba K, Yamazoe Y. Inhibitory potential of herbal medicines on human cytochrome P450-mediated oxidation: properties of umbelliferous or citrus crude drugs and their relative prescriptions. Jpn J Pharmacol 2001; 85: 399-408.

35 Wang Z, Su Z, Li C, Zheng H, Tan M, Wang Z. Textual study and resource investigation on traditional Chinese drugs duhuo, jiuyanduhuo and qianghuo. Zhongguo Zhong Yao Za Zhi 1995; 20: 515-7.

36 Sheng YF. Treatment of chronic Brucellosis with combined traditional Chinese medicine and Western medicine therapy. Zhongguo Zhong Xi Yi Jie He Za Zhi 1993; 13: 88-9.

37 Tanaka O, Yasuda Y, Yamasaki K, Mihashi S. Comparative study on the constituents of some Aralia species and notice of the original plants of crude drug “Duhuo”. Yakugaku Zasshi 1972; 92: 1058-60.

38 Shen X, Zheng W, Ma M, Zhang Z. The classification method of syndromes in the Bei ji qian jin yao fang (Essential Recipes for Emergent Use Worth A Thousand Gold). Zhonghua Yi Shi Za Zhi 2014; 44: 77-80.

39 Wang JY, Chen WM, Wen CS, Hung SC, Chen PW, Chiu JH. Du-Huo-JiSheng-Tang and its active component Ligusticum chuanxiong promote osteogenic differentiation and decrease the aging process of human mesenchymal stem cells. J Ethnopharmacol 2017; 198: 64-72. 
40 Chen Y, Li J, Li Q, Wang T, Xing L, Xu H, et al. Du-Huo-Ji-ShengTang attenuates inflammation of TNF-Tg mice related to promoting lymphatic drainage function. Evid Based Complement Alternat Med 2016; 2016: 7067691.

41 Chinese Pharmacopeia Commission. Pharmacopoeia of the People's Republic of China. 2010.

42 Pfirrmann CW, Metzdorf A, Zanetti M, Hodler J, Boos N. Magnetic resonance classification of lumbar intervertebral disc degeneration. Spine (Phila Pa 1976 ) 2001; 26: 1873-8.

43 Risbud MV, Shapiro IM. Role of cytokines in intervertebral disc degeneration: pain and disc content. Nat Rev Rheumatol 2014; 10 : 44-56.

44 Johnson ZI, Schoepflin ZR, Choi H, Shapiro IM, Risbud MV. Disc in flames: roles of TNF-alpha and IL-1beta in intervertebral disc degeneration. Eur Cell Mater 2015; 30: 104-16.

45 Palomino DC, Marti LC. Chemokines and immunity. Einstein (Sao Paulo) 2015; 13: 469-73.

46 Korhonen T, Karppinen J, Paimela L, Malmivaara A, Lindgren KA, Bowman $\mathrm{C}$, et al. The treatment of disc-herniation-induced sciatica with infliximab: one-year follow-up results of FIRST II, a randomized controlled trial. Spine (Phila Pa 1976 ) 2006; 31: 2759-66.

47 Walsh AJ, O'neill CW, Lotz JC. Glucosamine $\mathrm{HCl}$ alters production of inflammatory mediators by rat intervertebral disc cells in vitro. Spine J 2007; 7: 601-8.

48 Kim HR, Kim KW, Kim BM, Jung HG, Cho ML, Lee SH. Reciprocal activation of $\mathrm{CD} 4^{+} \mathrm{T}$ cells and synovial fibroblasts by stromal cellderived factor 1 promotes RANKL expression and osteoclastogenesis in rheumatoid arthritis. Arthritis Rheumatol 2014; 66: 538-48.

49 Zhang H, Zhang L, Chen L, Li W, Li F, Chen Q. Stromal cell-derived factor-1 and its receptor CXCR4 are upregulated expression in degenerated intervertebral discs. Int J Med Sci 2014; 11: 240-5.

50 Noort AR, van Zoest KP, Weijers EM, Koolwijk P, Maracle CX, Novack DV, et al. NF-kappaB-inducing kinase is a key regulator of inflammation-induced and tumour-associated angiogenesis. J Pathol 2014; 234: 375-85.

51 Tak PP, Firestein GS. NF-kappaB: a key role in inflammatory diseases. J Clin Invest 2001; 107: 7-11.

52 Ma Y, Cui J, Huang M, Meng K, Zhao Y. Effects of Duhuojisheng Tang and combined therapies on prolapse of lumbar intervertebral disc: a systematic review of randomized control trails. J Tradit Chin Med 2013; 33: 145-55.

53 Chen CW, Sun J, Li YM, Shen PA, Chen YQ. Action mechanisms of du-huo-ji-sheng-tang on cartilage degradation in a rabbit model of osteoarthritis. Evid Based Complement Alternat Med 2011; 2011: 571479.

54 Wang XL, Shang X, Cui Y, Zhao X, Zhang Y, Xie ML. Osthole inhibits inflammatory cytokine release through PPARalpha/gammamediated mechanisms in LPS-stimulated 3T3-L1 adipocytes. Immunopharmacol Immunotoxicol 2015; 37: 185-92.

$55 \mathrm{Wu}$ SJ. Osthole attenuates inflammatory responses and regulates the expression of inflammatory mediators in HepG2 cells grown in differentiated medium from 3T3-L1 preadipocytes. J Med Food 2015; 18: $972-9$.
56 Zhai YK, Pan YL, Niu YB, Li CR, Wu XL, Fan WT et al. The importance of the prenyl group in the activities of osthole in enhancing bone formation and inhibiting bone resorption in vitro. Int J Endocrinol 2014; 2014: 921954.

57 Tang DZ, Hou W, Zhou Q, Zhang M, Holz J, Sheu TJ et al. Osthole stimulates osteoblast differentiation and bone formation by activation of beta-catenin-BMP signaling. J Bone Miner Res 2010; 25: $1234-$ 45.

58 Wenjin C, Jianwei W. Protective effect of gentianine, a compound from Du Huo Ji Sheng Tang, against Freund's complete adjuvantinduced arthritis in rats. Inflammation 2017; 40: 1401-8.

59 Cao XF, Zhou I. HPLC determination of osthole in Duhuojisheng pills. China Pract Med 2010; 5: 36-7.

60 Wu L, Wang A, Geng H, Tian J, Liu H. Determination of paeoniflorin in Duhuojisheng mixture by HPLC-ELSD. China Pharmacist 2005; 8: 122-3.

61 Chen L, Liu JC, Zhang XN, Guo YY, Xu ZH, Cao W, et al. Downregulation of $N R 2 B$ receptors partially contributes to analgesic effects of Gentiopicroside in persistent inflammatory pain. Neuropharmacology 2008; 54: 1175-81.

62 Sozanski T, Kucharska AZ, Szumny A, Magdalan J, Bielska K, MerwidLad A, et al. The protective effect of the Cornus mas fruits (cornelian cherry) on hypertriglyceridemia and atherosclerosis through PPARalpha activation in hypercholesterolemic rabbits. Phytomedicine 2014; 21: 1774-84.

63 Sozanski T, Kucharska AZ, Rapak A, Szumny D, Trocha M, MerwidLad A, et al. Iridoid-loganic acid versus anthocyanins from the Cornus mas fruits (cornelian cherry): Common and different effects on diet-induced atherosclerosis, PPARs expression and inflammation. Atherosclerosis 2016; 254: 151-60.

64 Huang XT, Wang B, Zhang WH, Peng MQ, Lin D. Total glucosides of paeony suppresses experimental autoimmune uveitis in association with inhibition of Th1 and Th2 cell function in mice. Int J Immunopathol Pharmacol 2018; 32: 394632017751547.

65 Zhang W, Zhao L, Su SQ, Xu XX, Wu YG. Total glucosides of paeony attenuate renal tubulointerstitial injury in STZ-induced diabetic rats: role of Toll-like receptor 2. J Pharmacol Sci 2014; 125: 59-67.

66 Zhang W, Dai SM. Mechanisms involved in the therapeutic effects of Paeonia lactiflora Pallas in rheumatoid arthritis. Int Immunopharmacol 2012; 14: 27-31.

67 Wang S, Gao Z, Chen X, Lian X, Zhu H, Zheng J, et al. The anticoagulant ability of ferulic acid and its applications for improving the blood compatibility of silk fibroin. Biomed Mater 2008; 3: 044106.

68 Park SH, Kim SK, Shin IH, Kim HG, Choe JY. Effects of AIF on knee osteoarthritis patients: double-blind, randomized placebo-controlled study. Korean J Physiol Pharmacol 2009; 13: 33-7.

69 Duan DD, Wang Z, Zhang BL, Wang YY. Fangjiomics: revealing adaptive omics pharmacological mechanisms of the myriad combination therapies to achieve personalized medicine. Acta Pharmacol Sin 2015; 36: 651-3.

70 Wang Z, Duan DD, Wang YY. Editorial: Combination therapy of vascular diseases and fangjiomics: when west meets east in the era of phenomics. Curr Vasc Pharmacol 2015; 13: 420-2. 